\title{
Interval estimation for systems with time delays and algebraic constraints
}

\author{
Denis Efimov, Andrey Polyakov, Jean-Pierre Richard
}

\begin{abstract}
The problem of interval observer design is addressed for a class of descriptor linear systems with delays. Two sets of conditions are proposed. First, an interval observation for any input in the system is provided. Second, the control input is designed together with the observer gains in order to guarantee interval estimation and stabilization simultaneously. Efficiency of the proposed approach is illustrated by numerical experiments.
\end{abstract}

\section{INTRODUCTION}

The state estimation problem for uncertain models of industrial plants or biological systems has a great practical importance [1], [2], [3]. The model uncertainty can be presented by unknown (possibly time-varying) parameters, external disturbances and/or measurement noises. In such a way the designed estimator has to ensure a certain robustness of generated estimates with respect to perturbations. Another issue is that the observer design is structurally complicated in this case, since all uncertain terms should be either estimated simultaneously or avoided in the observer equations (e.g. substituted by some known bounds).

Then an important characteristic appears dealing with accuracy of the generated estimates of the state in the presence of all perturbations (unknown parameters, exogenous disturbances, measurement noise). The problem of accuracy evaluation is partially related with the problem of quantitative estimation of robustness. The difference is that usually for robustness quantification a gain should be computed between the maximal amplitude of a perturbation and the maximal amplitude of the response (the state estimation error in our case), while for accuracy evaluation the deviations from the real values have to be computed as tight as possible. It is strongly appreciated in applications to estimate this accuracy either off-line (during the design phase) or on-line using some numerical routines.

This work is supported in part by EU Interreg IV A 2 Mers Seas Zeeën Cross-border Cooperation Programme under SYSIASS project 06-020, by the Ministry of Higher Education and Research, Nord-Pas de Calais Regional Council and FEDER through the "Contrat de Projets Etat Region (CPER) CIA 2007-2013", by the Government of Russian Federation (Grant 074-U01) and the Ministry of Education and Science of Russian Federation (Project 14.Z50.31.0031).

The authors are with the Non-A team at Inria Lille, Parc Scientifique de la Haute Borne, 40 avenue Halley, 59650 Villeneuve d'Ascq, France and with LAGIS UMR 8219, Ecole Centrale de Lille, Avenue Paul Langevin, 59651 Villeneuve d'Ascq, France, \{denis.efimov; andrey.polyakov; jeanpierre.richard\}@inria.fr. The first author is with the Department of Control Systems and Informatics, National Research University ITMO, 49 avenue Kronverkskiy, 197101 Saint Petersburg, Russia.
There exist many approaches to design state observers for uncertain systems [1], [2], [3], all of them are heavily related with the type of the plant model. A special class of models is composed by the so-called (linear) continuous-time descriptor systems, singular systems or differential-algebraic systems [4]. Descriptor systems have attracted much attention due to the numerous applications in economics (Leontief dynamic model) [5], in electrical engineering [6], mechanical systems with constraints [7] or flow optimal control [8]. Another important class of models is described by timedelay differential equations [9]. The problem of observer design for delayed systems is rather complex [10], as well as the stability conditions for analysis of functional differential equations are rather complicated [11], [12]. Especially the observer synthesis is problematical for the cases when the model of a delayed system contains parametric and signal uncertainties, or when the delay is time-varying or uncertain [13], [14], [15], [16], [17].

The present work deals with an intersection of these classes, with linear descriptor time-delay systems. An observer solution for this more complex situation may be demanded in many real-world applications (economics, electrical circuits, flow control systems, and so on). Inclusion in a descriptor model of (almost always presented) delay effects increases accuracy of modeling (among others, in the case of convection effects in a fluid flow). In addition, a delayed descriptor system is a combination of two dynamics: a differential equation with a difference equation (in the delay-free case, algebraic constraint is static), which enlarges the class of physical phenomenon that can be modeled by the approach.

The solution of estimation problem for these systems is based on the set-membership estimation approaches [18], [19], [20], and in particular a design of interval observers [21], [22], [18], [23], [24], [25] is presented in this work. Some results have been obtained recently on synthesis of interval observers for time-delay systems [16], [17], and the objective is to extend these design tools to the class of descriptor systems. The advantages of the interval observers are that they are well adapted for observer design for highly uncertain systems (if the intervals of admissible values for unknown terms are given) and that they are capable to provide asymptotically rather tight bounds on the estimation accuracy, since the interval of admissible values for the state at each instant of time is evaluated.

The outline of this work is as follows. Some preliminary 
results are given in Section 2. Problem statement is presented in Section 3. Main results are formulated in Section 4. Numerical experiments and concluding remarks are presented in sections 5 and 6 respectively.

\section{Preliminaries}

In the rest of the paper, the following definitions will be used:

- $\mathbb{R}$ is the set of all real numbers $\left(\mathbb{R}_{+}=\{\tau \in \mathbb{R}: \tau \geq\right.$ $0\}), \mathcal{C}_{\tau}=C([-\tau, 0], \mathbb{R})$ is the set of continuous maps from $[-\tau, 0]$ into $\mathbb{R} ; \mathcal{C}_{\tau+}=\left\{y \in \mathcal{C}_{\tau}: y(s) \in \mathbb{R}_{+}, s \in\right.$ $[-\tau, 0]\}$;

- $x_{t}$ is an element of $\mathcal{C}_{\tau}^{n}$ associated with a map $x_{t}: \mathbb{R} \rightarrow$ $\mathbb{R}^{n}$ by $x_{t}(s)=x(t+s)$, for all $s \in[-\tau, 0]$;

- $|x|$ denotes the absolute value of $x \in \mathbb{R},\|x\|$ is the Euclidean norm of a vector $x \in \mathbb{R}^{n},\|\varphi\|=$ $\sup _{t \in[-\tau, 0]}\|\varphi(t)\|$ for $\varphi \in \mathcal{C}_{\tau}^{n},\|A\|$ corresponds to the Euclidean induced norm for a matrix $A \in \mathbb{R}^{n \times n}$;

- for a measurable and locally essentially bounded input $u: \mathbb{R}_{+} \rightarrow \mathbb{R}^{p}$ the symbol $\|u\|_{\left[t_{0}, t_{1}\right]}$ denotes its $L_{\infty}$ norm $\|u\|_{\left[t_{0}, t_{1}\right]}=e s s \sup \left\{\|u(t)\|, t \in\left[t_{0}, t_{1}\right]\right\}$, the set of all such inputs $u$ with the property $\|u\|_{[0,+\infty)}<\infty$ will be denoted as $\mathcal{L}_{\infty}^{p}$;

- for a matrix $A \in \mathbb{R}^{n \times n}$ the vector of its eigenvalues is denoted as $\lambda(A)$;

- $1_{n} \in \mathbb{R}^{n}$ is stated for a vector with unit elements, $I_{n}$ denotes the identity matrix of dimension $n \times n$;

- $a \mathcal{R} b$ corresponds to an elementwise relation $\mathcal{R}$ ( $a$ and $b$ are vectors or matrices): for example $a<b$ (vectors) means $\forall i: a_{i}<b_{i}$; for $\phi, \varphi \in \mathcal{C}_{\tau}^{n}$ the relation $\phi \mathcal{R} \varphi$ has to be understood elementwise for all domain of definition of the functions, i.e. $\phi(s) \mathcal{R} \varphi(s)$ for all $s \in[-\tau, 0]$

- the relation $P \prec 0(P \succ 0)$ means that the matrix $P \in$ $\mathbb{R}^{n \times n}$ is negative (positive) definite.

\section{A. Descriptor linear systems}

A descriptor system

$$
E \dot{x}(t)=A_{0} x(t)+B u(t),
$$

where $x \in \mathbb{R}^{n}$ and $u(t) \in \mathcal{L}_{\infty}^{m}$ are the state and the input (the matrices $E \in \mathbb{R}^{n \times n}, A_{0} \in \mathbb{R}^{n \times n}$ and $B \in \mathbb{R}^{n \times m}$ ), is called regular if the characteristic polynomial $\operatorname{det}(s E-A)$ does not vanish identically for all $s \in \mathbb{C}$ [26], [27]. In the regular case there exist matrices $P, Q \in \mathbb{R}^{n \times n}$ such that

$$
Q E P=\left[\begin{array}{cc}
I_{n_{1}} & 0 \\
0 & N
\end{array}\right], Q A_{0} P=\left[\begin{array}{cc}
J & 0 \\
0 & I_{n_{2}}
\end{array}\right],
$$

where $n_{2}=n-n_{1}$ for some $1 \leq n_{1}<n, N \in \mathbb{R}^{n_{2} \times n_{2}}$ and $J \in \mathbb{R}^{n_{1} \times n_{1}}$ are in the Jordan canonical form, the matrix $N$ is nilpotent of index $\nu$ (i.e. $N^{\nu}=0$ and $N^{\nu-1} \neq 0$ ). The descriptor system (or the pair $\left(E, A_{0}\right)$ ) has index that is the index of nilpotence $\nu$ of $N$. If a descriptor system has index $\nu>1$, then it admits impulsive solutions.

\section{B. Descriptor linear time-delay systems}

A descriptor time-delay system

$$
E \dot{x}(t)=A_{0} x(t)+A_{1} x(t-\tau)+B u(t),
$$

where $\tau>0$ is the delay and $x(t) \in \mathbb{R}^{n}$ is the state, $x_{0} \in \mathcal{C}_{\tau}^{n}$ is the initial condition, $u(t) \in \mathcal{L}_{\infty}^{m}$, has an index that is equal to the index of the pair $\left(E, A_{0}\right)$. If $\nu>1$ then the descriptor time-delay system has impulsive solutions. If a regular descriptor system has index 1 , then it can be presented in a canonical form (similar case has been considered in [26]), where

$$
\begin{gathered}
E=\left[\begin{array}{cc}
I_{n_{1}} & 0 \\
0 & 0
\end{array}\right] ; B=\left[\begin{array}{l}
B_{1} \\
B_{2}
\end{array}\right] ; \\
A_{i}=\left[\begin{array}{cc}
A_{i 1} & A_{i 2} \\
A_{i 3} & A_{i 4}
\end{array}\right], i=0,1 .
\end{gathered}
$$

Denote in this case $x=\left[\begin{array}{ll}x_{1}^{\mathrm{T}} & x_{2}^{\mathrm{T}}\end{array}\right]^{\mathrm{T}}$, where $x_{1} \in \mathbb{R}^{n_{1}}$ and $x_{2} \in \mathbb{R}^{n_{2}}$.

Proposition 1. [26] Assume that $\operatorname{det}\left(A_{04}\right) \neq 0$ and $x_{0} \in \mathcal{C}_{\tau}^{n}$, then for any $u(t) \in \mathcal{L}_{\infty}^{m}$ an absolute continuous solution of (1) exists for all $t \in \mathbb{R}_{+}$and it is unique.

Proposition 2. [26] Assume that $\operatorname{det}\left(A_{04}\right) \neq 0$ and $\max _{1 \leq i \leq n_{2}}\left|\lambda_{i}\left(A_{04}^{-1} A_{14}\right)\right|<1$. If there exist $P \in \mathbb{R}^{n \times n}$,

$$
\begin{gathered}
P=\left[\begin{array}{cc}
P_{1} & 0 \\
P_{2} & P_{3}
\end{array}\right], P_{1}=P_{1}^{T} \succ 0, \\
P_{1} \in \mathbb{R}^{n_{1} \times n_{1}}, P_{3} \in \mathbb{R}^{n_{2} \times n_{2}},
\end{gathered}
$$

and $U=U^{T} \in \mathbb{R}^{n \times n}$ that satisfy the following LMI for some $\gamma>0$ :

$$
\begin{gathered}
{\left[\begin{array}{ccc}
\Psi & P^{T} B & P^{T} A_{1} \\
B^{T} P & -\gamma^{2} I_{m} & 0 \\
A_{1}^{T} P & 0 & -U
\end{array}\right] \prec 0,} \\
\Psi=P^{T} A_{0}+A_{0}^{T} P+U,
\end{gathered}
$$

then the system (1), (2) is globally asymptotically stable for $u \equiv 0$ and any delay $\tau>0$, in addition its $H_{\infty}$ gain from the input $u$ to the state $x$ is less than $\gamma$.

\section{Positive descriptor linear time-delay systems}

Positive (nonnegative) or cooperative (monotone) models [28], [29], [30], [31] have a large area of applications for description the systems of different nature in biology, economics, chemistry etc.

Definition 1. A regular descriptor system (1) with index 1 is called positive if the restrictions $x_{0} \in \mathcal{C}_{\tau+}^{n}, u(t) \in \mathbb{R}_{+}^{m}$ for all $t \geq 0$ imply that $x(t) \in \mathbb{R}_{+}^{n}$ for all $t \geq 0$.

The generic results on positivity of the descriptor linear systems (without time delays) and their stability results are presented in [27], [32]. In the regular case with index 1 they can be seriously simplified [32]. In the spirit of these results, for time delay systems the following extensions can be obtained. For this purpose recall that a matrix $A$ is called 
Metzler if all its off-diagonal elements are nonnegative, a matrix $A$ is called nonnegative if $A \geq 0$ (elementwise).

Proposition 3. A regular descriptor system (1) with index 1 , written in the canonical form (2) with $\operatorname{det}\left(A_{04}\right) \neq 0$, is positive if and only if $A_{0}$ is Metzler and $A_{1}, B$ are nonnegative.

All proofs are omitted due to space limitations.

\section{Interval arithmetic}

The following lemmas have been proven in [22], [21]. Given a matrix $A \in \mathbb{R}^{m \times n}$, define $A^{+}=\max \{0, A\}$, $A^{-}=A^{+}-A$ (similarly for vectors) and denote the matrix of absolute values of all elements by $|A|=A^{+}+A^{-}$.

Lemma 1. Let $x \in \mathbb{R}^{n}$ be a vector variable, $\underline{x} \leq x \leq \bar{x}$ for some $\underline{x}, \bar{x} \in \mathbb{R}^{n}$, and $A \in \mathbb{R}^{m \times n}$ be a constant matrix, then

$$
A^{+} \underline{x}-A^{-} \bar{x} \leq A x \leq A^{+} \bar{x}-A^{-} \underline{x} .
$$

Lemma 2. Let $\underline{A} \leq A \leq \bar{A}$ for some $\underline{A}, \bar{A}, A \in \mathbb{R}^{n \times n}$ and $\underline{x} \leq x \leq \bar{x}$ for some $\underline{x}, \bar{x}, x \in \mathbb{R}^{n}$, then

$$
\begin{gathered}
\underline{A}^{+} \underline{x}^{+}-\bar{A}^{+} \underline{x}^{-}-\underline{A}^{-} \bar{x}^{+}+\bar{A}-\bar{x}^{-} \leq A x \\
\leq \bar{A}^{+} \bar{x}^{+}-\underline{A}^{+} \bar{x}^{-}-\bar{A} \underline{x}^{+}+\underline{A}^{-} \underline{x}^{-} .
\end{gathered}
$$

\section{Problem STATEMENT}

In this work we will consider an extended version of the descriptor time-delay system (1)

$$
\begin{aligned}
E \dot{x}(t) & =A_{0} x(t)+A_{1} x(t-\tau)+B u(t)+d(t), \\
y(t) & =C x(t)+v(t),
\end{aligned}
$$

where as before $\tau>0$ is the delay and $x(t) \in \mathbb{R}^{n}$ is the state (with initial conditions $\left.x_{0} \in \mathcal{C}_{\tau}^{n}\right) ; u(t) \in \mathcal{L}_{\infty}^{m}$ is the control signal; $d(t) \in \mathcal{L}_{\infty}^{n}$ is a disturbance; $y \in \mathbb{R}^{p}$ is the output signal available for measurements; $v(t) \in \mathcal{L}_{\infty}^{p}$ is the measurement noise. The constant matrices $E, A_{0}, A_{1}, B$ and $C$ have appropriate dimensions. The following restrictions are imposed on (4). The case of multiple delays can be incorporated using the results of [26].

Assumption 1. The system (4) is regular with index 1, $\operatorname{det}\left(A_{04}\right) \neq 0$, presented in the canonical form (2).

Assumption 2. The state $x(t)$ is a bounded function of time (i.e. $\left.x(t) \in \mathcal{L}_{\infty}^{n}\right)$.

Assumption 3. There is a known constant $V>0$ such that $\left\|v_{i}\right\|_{[0, \infty)} \leq V$ for all $1 \leq i \leq p$, and there exist two known functions $\underline{d}, \bar{d}: \mathbb{R}_{+} \rightarrow \mathbb{R}^{n}, \underline{d}(t), \bar{d}(t) \in \mathcal{L}_{\infty}^{n}$ such that $\underline{d}(t) \leq$ $d(t) \leq \bar{d}(\bar{t})$ for all $t \geq 0$.

The first assumption claims that the system (4) is regular and it has no impulsive solutions. It is also indicated that (4) is transformed to the canonical representation (that is always possible for regular systems with index 1). In the second assumption, boundedness of the state is imposed, which is a typical restriction in the estimation theory. In the third assumption it is assumed that the noise is bounded by $V$ and the lower and upper bounds for $d(t)$ are given (an interval of admissible values for $d(t)$ at each instant of time $t \geq 0$ ).

The objective is to design an interval observer for (4), i.e. a dynamical system

$$
\begin{gathered}
\dot{z}(t)=F\left(z_{t}, y_{t}, u(t), V, \underline{d}(t), \bar{d}(t)\right), z_{0} \in \mathcal{C}_{\tau}^{k}, k>0, \\
\underline{x}(t)=\underline{H}\left(z_{t}, y_{t}, u(t), V, \underline{d}(t), \bar{d}(t)\right), \\
\bar{x}(t)=\bar{H}\left(z_{t}, y_{t}, u(t), V, \underline{d}(t), \bar{d}(t)\right),
\end{gathered}
$$

such that $\underline{x}(t) \leq x(t) \leq \bar{x}(t)$ for all $t \geq 0$ provided that $\underline{x}_{0} \leq x_{0} \leq \bar{x}_{0}$. In addition, if $\lim _{t \rightarrow+\infty} \mid \underline{d}(t)-$ $\bar{d}(t)\left|=\lim _{t \rightarrow+\infty}\right| v(t) \mid=0$, then $\lim _{t \rightarrow+\infty}|\underline{x}(t)-\bar{x}(t)|=$ $\lim _{t \rightarrow+\infty}|x(t)-\bar{x}(t)|=0$.

\section{MAIN RESULTS}

First of all note that for any $L_{1}, L_{2} \in \mathbb{R}^{n \times p}$ the system (4) can be rewritten as follows:

$$
\begin{aligned}
E \dot{x}(t)= & A_{0} x(t)+A_{1} x(t-\tau)+B u(t)+d(t) \\
& +L_{1}(y(t)-C x(t)-v(t)) \\
& +L_{2}(y(t-\tau)-C x(t-\tau)-v(t-\tau)) \\
= & \left(A_{0}-L_{1} C\right) x(t)+\left(A_{1}-L_{2} C\right) x(t-\tau) \\
& +B u(t)+\delta(t)+L_{1} y(t)+L_{2} y(t-\tau),
\end{aligned}
$$

where $\delta(t)=d(t)-L_{1} v(t)-L_{2} v(t-\tau)$, and using the result of Lemma 2 , for all $t \geq 0$ :

$$
\begin{gathered}
\underline{\delta}(t) \leq \delta(t) \leq \bar{\delta}(t), \\
\underline{\delta}(t)=\underline{d}(t)-\left(\left|L_{1}\right|+\left|L_{2}\right|\right) 1_{p} V, \\
\bar{\delta}(t)=\bar{d}(t)+\left(\left|L_{1}\right|+\left|L_{2}\right|\right) 1_{p} V .
\end{gathered}
$$

Below, two sets of different conditions on $L_{1}$ and $L_{2}$ are considered with two different interval observers. Next, we will skip the requirement of Assumption 2 that the state $x(t)$ is bounded, and in addition to $L_{1}, L_{2}$ we will design a control $u(t)$ dependent on the interval estimates $\underline{x}(t), \bar{x}(t)$ providing the state $x(t)$ stabilization, as it has been done in [21], [33] for ordinary differential equations and time-delay systems (with the delay in the input channel only).

Further, denote

$$
C=\left[\begin{array}{ll}
C_{1} & C_{2}
\end{array}\right], L_{i}=\left[\begin{array}{c}
L_{i 1} \\
L_{i 2}
\end{array}\right], i=1,2,
$$

where $C_{1} \in \mathbb{R}^{p \times n_{1}}, C_{2} \in \mathbb{R}^{p \times n_{2}}, L_{i 1} \in \mathbb{R}^{n_{1} \times p}$ and $L_{i 2} \in$ $\mathbb{R}^{n_{2} \times p}$.

\section{A. The case of a positive system}

Actually we will not assume explicitly in this subsection that $x(t) \in \mathbb{R}_{+}^{n}$ and that (4) is positive, but the conditions imposed on $L_{1}$ and $L_{2}$ imply that the linear part in (5) is positive. 
Consider the following interval observer:

$$
\begin{aligned}
E \underline{\dot{x}}(t)= & \left(A_{0}-L_{1} C\right) \underline{x}(t)+\left(A_{1}-L_{2} C\right) \underline{x}(t-\tau) \\
& +B u(t)+\underline{\delta}(t)+L_{1} y(t)+L_{2} y(t-\tau), \\
E \dot{\bar{x}}(t)= & \left(A_{0}-L_{1} C\right) \bar{x}(t)+\left(A_{1}-L_{2} C\right) \bar{x}(t-\tau) \\
& +B u(t)+\bar{\delta}(t)+L_{1} y(t)+L_{2} y(t-\tau) .
\end{aligned}
$$

As we can conclude from (6), the dynamics of $\underline{x}(t)$ and $\bar{x}(t)$ are independent.

Theorem 1. Let assumptions $1-3$ be satisfied and $A_{0}-L_{1} C$, $A_{1}-L_{2} C$ be a Metzler and a nonnegative matrix respectively with $\operatorname{det}\left(A_{04}-L_{12} C_{2}\right) \neq 0$. Then in (4), (6), $\underline{x}(t) \leq$ $x(t) \leq \bar{x}(t)$ for all $t \geq 0$ provided that $\underline{x}_{0} \leq x_{0} \leq \bar{x}_{0}$. If $\max _{1 \leq i \leq n_{2}}\left|\lambda_{i}\left(\left[A_{04}-L_{12} C_{2}\right]^{-1}\left[A_{14}-L_{22} C_{2}\right]\right)\right|<1$ and there exist matrices $P \in \mathbb{R}^{n \times n}$ of the form (3) and $U=U^{T} \in \mathbb{R}^{n \times n}$ that satisfy the LMI

$$
\begin{gathered}
{\left[\begin{array}{ccc}
\Psi & P^{T} & P^{T}\left(A_{1}-L_{2} C\right) \\
P & -\gamma^{2} I_{n} & 0 \\
\left(A_{1}-L_{2} C\right)^{T} P & 0 & -U
\end{array}\right] \prec 0,} \\
\Psi=P^{T}\left(A_{0}-L_{1} C\right)+\left(A_{0}-L_{1} C\right)^{T} P+U
\end{gathered}
$$

for some $\gamma>0$, then $\underline{x}(t), \bar{x}(t) \in \mathcal{L}_{\infty}^{n}$ for any $\tau>0$ and an $H_{\infty}$ gain from the inputs $\delta-\underline{\delta}, \bar{\delta}-\delta$ to the state estimation errors $x-\underline{x}, \bar{x}-x$ respectively is less than $\gamma$.

Remark 1. Note that the result of Theorem 1 means that if $\delta(t)-\underline{\delta}(t) \rightarrow 0, \bar{\delta}(t)-\delta(t) \rightarrow 0$ with $t \rightarrow+\infty$, then $\underline{x}(t) \rightarrow x(t) \leftarrow \bar{x}(t)$ asymptotically.

\section{B. The case of a positive delay-free system}

In this part we would like to relax the restrictions introduced in the previous subsection for $L_{1}$ and $L_{2}$. For this purpose consider another interval observer:

$$
\begin{aligned}
E \underline{\dot{x}}(t)= & \left(A_{0}-L_{1} C\right) \underline{x}(t)+\left(A_{1}-L_{2} C\right)^{+} \underline{x}(t-\tau) \\
& -\left(A_{1}-L_{2} C\right)^{-} \bar{x}(t-\tau) \\
& +B u(t)+\underline{\delta}(t)+L_{1} y(t)+L_{2} y(t-\tau), \quad(7) \\
E \dot{\bar{x}}(t)= & \left(A_{0}-L_{1} C\right) \bar{x}(t)+\left(A_{1}-L_{2} C\right)^{+} \bar{x}(t-\tau) \\
& -\left(A_{1}-L_{2} C\right)^{-} \underline{x}(t-\tau) \\
& +B u(t)+\bar{\delta}(t)+L_{1} y(t)+L_{2} y(t-\tau) .
\end{aligned}
$$

In this case the dynamics of $\underline{x}, \bar{x}$ are interrelated in (7).

Theorem 2. Let assumptions $1-3$ be satisfied and $A_{0}-L_{1} C$ be a Metzler matrix with $\operatorname{det}\left(A_{04}-L_{12} C_{2}\right) \neq 0$. Then in (4), (7), $\underline{x}(t) \leq x(t) \leq \bar{x}(t)$ for all $t \geq 0$ provided that $\underline{x}_{0} \leq x_{0} \leq \bar{x}_{0}$. If $\max _{1 \leq i \leq 2 n_{2}}\left|\lambda_{i}\left(\Lambda_{04}^{-1} \Lambda_{14}\right)\right|<1$ and there exist matrices $P \in \mathbb{R}^{2 n \times 2 n}$,

$$
\begin{aligned}
& P=\left[\begin{array}{cc}
P_{1} & 0 \\
P_{2} & P_{3}
\end{array}\right], P_{1}=P_{1}^{T} \succ 0, \\
& P_{1} \in \mathbb{R}^{2 n_{1} \times 2 n_{1}}, P_{3} \in \mathbb{R}^{2 n_{2} \times 2 n_{2}}
\end{aligned}
$$

and $U=U^{T} \in \mathbb{R}^{2 n \times 2 n}$ that satisfy the LMI

$$
\begin{gathered}
{\left[\begin{array}{ccc}
\Psi & P^{T} & P^{T} \Lambda_{1} \\
P & -\gamma^{2} I_{2 n} & 0 \\
\Lambda_{1}^{T} P & 0 & -U
\end{array}\right] \prec 0,} \\
\Psi=P^{T} \Lambda_{0}+\Lambda_{0}^{T} P+U, \\
\Lambda_{i}=\left[\begin{array}{cc}
\Lambda_{i 1} & \Lambda_{i 2} \\
\Lambda_{i 3} & \Lambda_{i 4}
\end{array}\right], i=1,2, \\
\Lambda_{01}=\left[\begin{array}{cc}
A_{01}-L_{11} C_{1} & 0 \\
0 & A_{01}-L_{11} C_{1}
\end{array}\right], \\
\Lambda_{02}=\left[\begin{array}{cc}
A_{02}-L_{11} C_{2} & 0 \\
0 & A_{02}-L_{11} C_{2}
\end{array}\right], \\
\Lambda_{03}=\left[\begin{array}{cc}
A_{03}-L_{12} C_{1} & 0 \\
0 & A_{03}-L_{12} C_{1}
\end{array}\right], \\
\Lambda_{04}=\left[\begin{array}{cc}
A_{04}-L_{12} C_{2} & 0 \\
0 & A_{04}-L_{12} C_{2}
\end{array}\right], \\
\Lambda_{11}=\left[\begin{array}{ll}
\left(A_{11}-L_{21} C_{1}\right)^{+} & \left(A_{11}-L_{21} C_{1}\right)^{-} \\
\left(A_{11}-L_{21} C_{2}\right)^{-} & \left(A_{11}-L_{21} C_{1}\right)^{+}
\end{array}\right], \\
\Lambda_{12}=\left[\begin{array}{ll}
\left(A_{12}-L_{21} C_{2}\right)^{+} & \left(A_{12}-L_{21} C_{2}\right)^{-} \\
\left(A_{12}-L_{21} C_{2}\right)^{-} & \left(A_{12}-L_{21} C_{2}\right)^{+}
\end{array}\right], \\
\Lambda_{13}=\left[\begin{array}{ll}
\left(A_{13}-L_{22} C_{1}\right)^{+} & \left(A_{13}-L_{22} C_{1}\right)^{-} \\
\left(A_{13}-L_{22} C_{1}\right)^{-} & \left(A_{13}-L_{22} C_{1}\right)^{+}
\end{array}\right], \\
\Lambda_{14}=\left[\begin{array}{ll}
\left(A_{14}-L_{22} C_{2}\right)^{+} & \left(A_{14}-L_{22} C_{2}\right)^{-} \\
\left(A_{14}-L_{22} C_{2}\right)^{-} & \left(A_{14}-L_{22} C_{2}\right)^{+}
\end{array}\right],
\end{gathered}
$$

for some $\gamma>0$, then $\underline{x}(t), \bar{x}(t) \in \mathcal{L}_{\infty}^{n}$ for any $\tau>0$ and an $H_{\infty}$ gain from the inputs $\delta-\underline{\delta}, \bar{\delta}-\delta$ to the state estimation errors $x-\underline{x}, \bar{x}-x$ respectively is less than $\gamma$.

Remark 2. Contrarily Theorem 1 , the restriction $A_{1}-L_{2} C \geq$ 0 is not imposed in Theorem 2, the price for that is more complicated equations of interval observer (check (7) with respect to (6)) and more sophisticated matrix inequalities.

The conditions of Theorem 2 may be relaxed asking existence of a transformation matrix $S \in \mathbb{R}^{n_{1} \times n_{1}}$ such that $S^{-1}\left(A_{0}-L_{1} C\right) S$ is Metzler, the conditions of such a transformation matrix existence can be found in [25].

Remark 3. Finally note that a bounded function $d(\cdot)$ can be dependent on measured output $y$ and input $u$ in a nonlinear fashion, which does not change the applicability conditions of theorems 1 and 2 provided that $\underline{d}(t)$ and $\bar{d}(t)$ can be constructed. Thus the proposed interval observers (6) and (7) can be applied for estimation of uncertain nonlinear differential-algebraic systems. The delay $\tau$ can also be considered time-varying and uncertain under a mild modification of the structure of observers (6) and (7) in the way it has been proposed in [17].

C. Stabilization of descriptor time-delay systems using interval observer (6)

In this subsection we will try to skip Assumption 2 and, in order to simplify the presentation, we will consider the case 
of interval observer (6) only (similar results can be obtained for (7), they are omitted for compactness).

In Theorem 1 the gains $L_{1}$ and $L_{2}$ have been used to guarantee the properties of positivity and stability for the dynamics of estimation errors $\underline{e}(t), \bar{e}(t)$. The positivity property has been obtained uniformly in $u(t)$. Thus the control design can be applied in order to ensure boundedness of the observer estimates $\underline{x}(t), \bar{x}(t)$, that in its turn $(\underline{x}(t) \leq x(t) \leq \bar{x}(t)$ for all $t \geq 0$ ) will provide boundedness of $x(t)$. An advantage of this approach is that the system (4) is uncertain and the state of that system is not measured, while the observer (6) is a completely known system with the accessible state $\underline{x}(t), \bar{x}(t)$ [21]. A drawback is that the dimension of the state for (6) is $2 n$, while the dimension of the control is $m$, similarly to (4).

It is also necessary to take into account that for stabilization of $\underline{x}(t), \bar{x}(t)$ in (6), the signals $y(t)$ and $y(t-\tau)$ form a kind of functional perturbation, which is globally Lipschitz with respect to $\underline{x}(t), \bar{x}(t)$. Indeed, from the relation $\underline{x}(t) \leq x(t) \leq \bar{x}(t)$ we have

$$
\begin{aligned}
\left|x_{i}(t)\right| & \leq \max \left\{\left|\underline{x}_{i}(t)\right|,\left|\bar{x}_{i}(t)\right|\right\} \quad \forall 1 \leq i \leq n, \\
\left|x_{i}(t)\right|^{2} & \leq\left|\underline{x}_{i}(t)\right|^{2}+\left|\bar{x}_{i}(t)\right|^{2} \quad \forall 1 \leq i \leq n, \\
\|x(t)\| & \leq \sqrt{\|\underline{x}(t)\|^{2}+\|\bar{x}(t)\|^{2}} \\
& \leq \sqrt{2} \max \{\|\underline{x}(t)\|,\|\bar{x}(t)\|\},
\end{aligned}
$$

then $\|y(t)\| \leq\|C\| \sqrt{2} \max \{\|\underline{x}(t)\|,\|\bar{x}(t)\|\}$.

Take

$$
\begin{aligned}
u(t)= & \underline{K}_{1} \underline{x}(t)+\bar{K}_{1} \bar{x}(t)+\underline{K}_{2} \underline{x}(t-\tau) \\
& +\bar{K}_{2} \bar{x}(t-\tau)-R_{1} y(t)-R_{2} y(t-\tau)
\end{aligned}
$$

for some matrices $\underline{K}_{i}, \bar{K}_{i} \in \mathbb{R}^{m \times n}, R_{i} \in \mathbb{R}^{m \times p}$ with $i=$ 1,2 . The idea of introduction of $R_{1}$ and $R_{2}$ is to minimize the influence of $y(t), y(t-\tau)$ on the dynamics of $\underline{x}(t), \bar{x}(t)$ in (6), i.e. to minimize the norm of $F_{i}=L_{i}-B R_{i}$. In a similar way, a term can be introduced in $u(t)$ in order to reduce the influence of $\underline{\delta}(t), \bar{\delta}(t)$.

Theorem 3. Let assumptions 1,3 be satisfied and $A_{0}-L_{1} C$, $A_{1}-L_{2} C$ be a Metzler and a nonnegative matrix respectively with $\operatorname{det}\left(A_{04}-L_{12} C_{2}\right) \neq 0$. Then in (4), (6) with the control (9), $\underline{x}(t) \leq x(t) \leq \bar{x}(t)$ for all $t \geq 0$ provided that $\underline{x}_{0} \leq$ $x_{0} \leq \bar{x}_{0}$. If $\max _{1 \leq i \leq n_{2}}\left|\lambda_{i}\left(\Delta_{04}^{-1} \Delta_{14}\right)\right|<1$ and there exist matrices $P \in \mathbb{R}^{2 n \times 2 n}$ of the form (8) and $U=U^{T} \in \mathbb{R}^{2 n \times 2 n}$ that satisfy the matrix inequalities

$$
\begin{gathered}
{\left[\begin{array}{ccc}
\Psi & P^{T} & P^{T} \Delta_{1} \\
P & -\gamma^{2} I_{n} & 0 \\
\Delta_{1}^{T} P & 0 & -U
\end{array}\right] \prec 0,} \\
\Psi=P^{T} \Delta_{0}+\Delta_{0}^{T} P+U, \\
\Delta_{i}=\left[\begin{array}{cc}
\Delta_{i 1} & \Delta_{i 2} \\
\Delta_{i 3} & \Delta_{i 4}
\end{array}\right], i=1,2,
\end{gathered}
$$

$$
\begin{aligned}
\Delta_{01} & =\left[\begin{array}{cc}
A_{01}-L_{11} C_{1}+B_{1} \underline{K}_{11} & B_{1} \bar{K}_{11} \\
B_{1} \underline{K}_{11} & A_{01}-L_{11} C_{1}+B_{1} \bar{K}_{11}
\end{array}\right], \\
\Delta_{02} & =\left[\begin{array}{cc}
A_{02}-L_{11} C_{2}+B_{1} \underline{K}_{12} & B_{1} \bar{K}_{12} \\
B_{1} \underline{K}_{12} & A_{02}-L_{11} C_{2}+B_{1} \bar{K}_{12}
\end{array}\right], \\
\Delta_{03} & =\left[\begin{array}{cc}
A_{03}-L_{12} C_{1}+B_{2} \underline{K}_{11} & B_{2} \bar{K}_{11} \\
B_{2} \underline{K}_{11} & A_{03}-L_{12} C_{1}+B_{2} \bar{K}_{11}
\end{array}\right], \\
\Delta_{04} & =\left[\begin{array}{cc}
A_{04}-L_{12} C_{2}+B_{2} \underline{K}_{12} & B_{2} \bar{K}_{12} \\
B_{2} \underline{K}_{12} & A_{04}-L_{12} C_{2}+B_{2} \bar{K}_{12}
\end{array}\right], \\
\Delta_{11} & =\left[\begin{array}{cc}
A_{11}-L_{21} C_{1}+B_{1} \underline{K}_{21} & A_{11}-L_{21} C_{1}+B_{1} \bar{K}_{21}
\end{array}\right], \\
B_{1} \underline{K}_{21} & B_{1} \bar{K}_{22} \\
\Delta_{12} & =\left[\begin{array}{cc}
A_{12}-L_{21} C_{2}+B_{1} \underline{K}_{22} & A_{12}-L_{21} C_{2}+B_{1} \bar{K}_{22}
\end{array}\right], \\
B_{1} \underline{K}_{22} & B_{2} \bar{K}_{21} \\
\Delta_{13} & =\left[\begin{array}{cc}
A_{13}-L_{22} C_{1}+B_{2} \underline{K}_{21} & A_{13}-L_{22} C_{1}+B_{2} \bar{K}_{21}
\end{array}\right], \\
B_{2} \underline{K}_{21} & B_{2} \bar{K}_{22} \\
\Delta_{14} & =\left[\begin{array}{cc}
A_{14}-L_{22} C_{2}+B_{1} \underline{K}_{22} & A_{14}-L_{22} C_{2}+B_{2} \bar{K}_{22}
\end{array}\right] .
\end{aligned}
$$

for $\gamma<\left[\|C\| \sqrt{2}\left(\left\|F_{1}\right\|+\left\|F_{2}\right\|\right)\right]^{-1}$, then $x(t), \underline{x}(t), \bar{x}(t) \in$ $\mathcal{L}_{\infty}^{n}$ for any $\tau>0$ and an $H_{\infty}$ gain from the inputs $\underline{\delta}, \bar{\delta}$ to the states $x, \bar{x}$ is less than $\gamma$ and to the state $x$ is less than $\sqrt{2} \gamma$.

In order to calculate $L_{i}, \underline{K}_{i}, \bar{K}_{i}, i=1,2$ the conditions of Theorem 3 can be decoupled. First, asking for $L_{i}$ such that $A_{0}-L_{1} C$ is Metzler and Hurwitz and $A_{1}-L_{2} C$ is nonnegative and Schur stable. Second, looking for $K_{i}$, $\bar{K}_{i}$ such that the LMI in the formulation of Theorem 3 is satisfied.

\section{Simulations}

Let us consider system (4) with

$$
\begin{gathered}
A_{0}=\left[\begin{array}{ccc}
-2 & 0 & 1 \\
-1 & -4 & 0 \\
-0.5 & 1 & -1
\end{array}\right], A_{1}=\left[\begin{array}{ccc}
0 & 0.5 & 0 \\
-1 & 0 & 0.25 \\
0 & 1 & 0.25
\end{array}\right], \\
E=\left[\begin{array}{lll}
1 & 0 & 0 \\
0 & 1 & 0 \\
0 & 0 & 0
\end{array}\right], B=\left[\begin{array}{l}
0 \\
1 \\
0
\end{array}\right], C=\left[\begin{array}{l}
1 \\
0 \\
0
\end{array}\right]^{\mathrm{T}}, \\
d(t)=\left[\begin{array}{c}
\sin \left(x_{1}\right)+\delta \sin \left(x_{2}\right) \\
\sin \left(x_{1}\right)-\delta \cos (2 t) \\
\sin \left(0.5 x_{1}\right)+\delta \sin \left(x_{2} t\right)
\end{array}\right], u(t)=\sin \left(\frac{t}{4}\right), \\
v(t)=V \sin (25 t), \delta=1.5, V=0.2, \tau=0.1,
\end{gathered}
$$

where the part of $d$ proportional to $\delta$ is unknown (the model uncertainty). The system is nonlinear since $d$ depends on $x$, however the known part of $d$ depends on $x_{1}(t)=y(t)-v(t)$, then we can design $\underline{d}(t)$ and $\bar{d}(t)$ dependent on the measured signals only. Take

$$
L_{1}=\left[\begin{array}{c}
3 \\
-1 \\
-0.5
\end{array}\right], L_{2}=\left[\begin{array}{c}
0 \\
-1 \\
0
\end{array}\right],
$$

then the LMI of Theorem 1 is satisfied for $\gamma=1, P=$ $\operatorname{diag}\left[\begin{array}{lll}0.05 & 1 & 0.3\end{array}\right]$ and $U=0.3 P$. All conditions of this theorem are valid and the results of interval observer (6) application are given in Fig. 1, for the coordinates $x_{2}$ and $x_{3}$. 

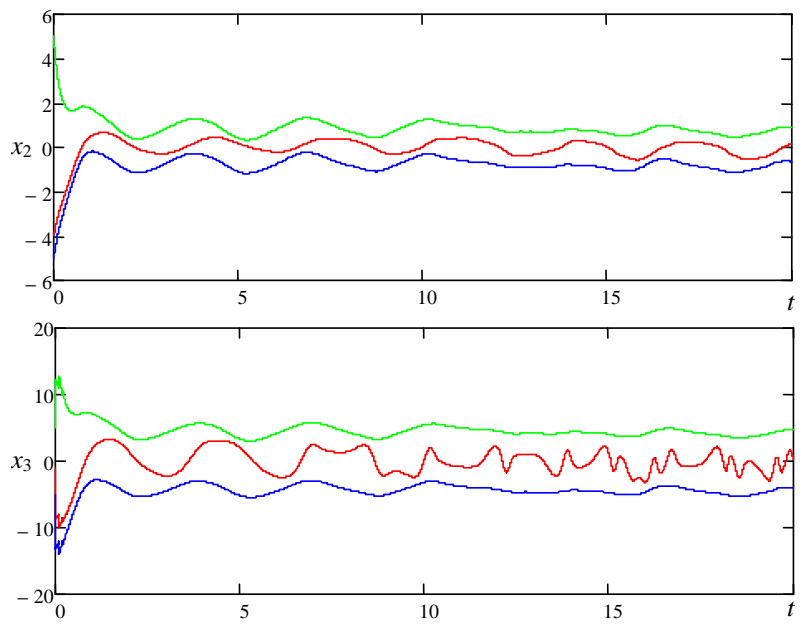

Figure 1. The results of simulation of the interval observer (6)

\section{CONCLUSION}

For a class of regular descriptor linear systems of index 1 with delays two interval observers are proposed. Two sets of conditions are developed for these observers guaranteeing an interval estimation in the presence of any control input. Next, a control input is designed based on interval estimates providing interval estimation and stabilization simultaneously. Efficiency of the proposed approach is demonstrated in simulation.

As directions of future works, the development of computationally simple LMIs and the analysis of a time-varying delay can be considered.

\section{REFERENCES}

[1] G. Besançon, ed., Nonlinear Observers and Applications, vol. 363 of Lecture Notes in Control and Information Sciences. Springer, 2007.

[2] T. Meurer, K. Graichen, and E.-D. Gilles, eds., Control and Observer Design for Nonlinear Finite and Infinite Dimensional Systems, vol. 322 of Lecture Notes in Control and Information Sciences. Springer, 2005.

[3] T. Fossen and H. Nijmeijer, New Directions in Nonlinear Observer Design. Springer, 1999.

[4] G.-R. Duan, Analysis and Design of Descriptor Linear Systems, vol. 23 of Advances in Mechanics and Mathematics. Springer, 2010.

[5] M. Silva and T. de Lima, "Looking for nonnegative solutions of a Leontief dynamic model," Linear Algebra, vol. 364, pp. 281-316, 2003.

[6] S. Campbell, Singular Systems of Differential Equations. London: Pitman, 1980

[7] R. Schüpphaus and P. Müller, "Control analysis and synthesis of linear mechanical descriptor systems," in Advanced Multibody System Dynamics (W. Schiehlen, ed.), vol. 20 of Solid Mechanics and Its Applications, pp. 463-468, Springer Netherlands, 1993.

[8] P. Losse, V. Mehrmann, L. Poppe, and T. Reis, "The modified optimal $H_{\infty}$ control problem for descriptor systems," SIAM Journal Control and Optimization, vol. 47, pp. 2795-2811, 2008.

[9] V. Kolmanovskii and A. Myshkis, Introduction to the Theory and Applications of Functional Differential Equations. Dordrecht: Kluwer Academic Publishers, 1999.

[10] R. Sipahi, S.-I. Niculescu, C. Abdallah, W. Michiels, and K. Gu, "Stability and stabilization of systems with time delay limitations and opportunities," IEEE Control Systems Magazine, vol. 31, no. 1, pp. 3865, 2011.
[11] J.-P. Richard, "Time delay systems: an overview of some recent advances and open problems," Automatica, vol. 39, no. 10, pp. 16671694, 2003.

[12] E. Fridman, "Descriptor discretized Lyapunov functional method: Analysis and design," IEEE Transactions on Automatic Control, vol. 51, no. 5, pp. 890-897, 2006.

[13] C. Briat, O. Sename, and J.-F. Lafay, "Design of LPV observers for LPV time-delay systems: an algebraic approach," Int. J. Control, vol. 84, no. 9, pp. 1533-1542, 2011.

[14] M. Darouach, "Linear functional observers for systems with delays in state variables," IEEE Transactions on Automatic Control, vol. 46, no. 3, pp. 491-496, 2001

[15] G. Zheng, J.-P. Barbot, D. Boutat, T. Floquet, and J.-P. Richard, "On observation of time-delay systems with unknown inputs," IEEE Trans. Automatic Control, vol. 56, no. 8, pp. 1973-1978, 2011.

[16] F. Mazenc, S. Niculescu, and O. Bernard, "Exponentially stable interval observers for linear systems with delay," SIAM J. Control Optim., vol. 50, no. 1, pp. 286-305, 2012.

[17] D. Efimov, W. Perruquetti, and J.-P. Richard, "Interval estimation for uncertain systems with time-varying delays," International Journal of Control, vol. 86, no. 10, pp. 1777-1787, 2013.

[18] J. Gouzé, A. Rapaport, and M. Hadj-Sadok, "Interval observers for uncertain biological systems," Ecological Modelling, vol. 133, pp. 46$56,2000$.

[19] L. Jaulin, "Nonlinear bounded-error state estimation of continuous time systems," Automatica, vol. 38, no. 2, pp. 1079-1082, 2002.

[20] M. Kieffer and E. Walter, "Guaranteed nonlinear state estimator for cooperative systems," Numerical Algorithms, vol. 37, pp. 187-198, 2004.

[21] D. Efimov, T. Raïssi, and A. Zolghadri, "Control of nonlinear and lpv systems: interval observer-based framework," IEEE Trans. Automatic Control, vol. 58, no. 3, pp. 773-782, 2013.

[22] D. Efimov, L. Fridman, T. Raïssi, A. Zolghadri, and R. Seydou, "Interval estimation for LPV systems applying high order sliding mode techniques," Automatica, vol. 48, pp. 2365-2371, 2012.

[23] F. Mazenc and O. Bernard, "Interval observers for linear time-invariant systems with disturbances," Automatica, vol. 47, no. 1, pp. 140-147, 2011.

[24] M. Moisan, O. Bernard, and J. Gouzé, "Near optimal interval observers bundle for uncertain bio-reactors," Automatica, vol. 45, no. 1, pp. 291295, 2009.

[25] T. Raïssi, D. Efimov, and A. Zolghadri, "Interval state estimation for a class of nonlinear systems," IEEE Trans. Automatic Control, vol. 57, no. 1, pp. 260-265, 2012

[26] E. Fridman and U. Shaked, " $H_{\infty}$ control of linear state-delay descriptor systems: an lmi approach," Linear Algebra and its Applications, vol. 351-352, pp. 271-302, 2002.

[27] E. Virnik, "Stability analysis of positive descriptor systems," Linear Algebra Appl., vol. 429, pp. 2640-2659, 2008.

[28] M. Dambrine and J.-P. Richard, "Stability analysis of time-delay systems," Dynamic Systems and Applications, vol. 2, pp. 405-414, 1993.

[29] H. Smith, Monotone Dynamical Systems: An Introduction to the Theory of Competitive and Cooperative Systems, vol. 41 of Surveys and Monographs. Providence: AMS, 1995.

[30] L. Farina and S. Rinaldi, Positive Linear Systems: Theory and Applications. New York: Wiley, 2000.

[31] W. Haddad and V. Chellaboina, "Stability theory for nonnegative and compartmental dynamical systems with time delay," Syst. Control Letters, vol. 51, pp. 355-361, 2004.

[32] E. Virnik, Analysis of positive descriptor systems. $\mathrm{PhD}$ thesis, $\mathrm{TU}$ Berlin, Berlin, 2008.

[33] A. Polyakov, D. Efimov, W. Perruquetti, and J.-P. Richard, "Output stabilization of time-varying input delay systems using interval observation technique," Automatica, vol. 49, no. 11, pp. 3402-3410, 2013. 